\title{
REM observations of the Herbig Ae stars V346 Ori and PDS2
}

\author{
S. Bernabei, ${ }^{1,2}$ M. Marconi, ${ }^{3}$ V. Ripepi, ${ }^{3}$ S. Leccia, ${ }^{3}$ E. Rodríguez, ${ }^{4}$ T. D. Oswalt, ${ }^{5}$
} M. J. López-González, ${ }^{4}$ F. J. Aceituno, ${ }^{4}$ A. Ruoppo, ${ }^{3,6}$ F. Palla, ${ }^{7}$ M. J. P. F. G. Monteiro, ${ }^{8}$ E. Molinari, ${ }^{9}$ G. Chincarini, ${ }^{9}$ F. M. Zerbi, ${ }^{9}$ S. Covino, ${ }^{9}$ V. Testa, ${ }^{10}$ G. Tosti, ${ }^{11}$ F. Vitali, ${ }^{10}$ L. A. Antonelli, ${ }^{10}$ P. Conconi, ${ }^{9}$ G. Malaspina, ${ }^{9}$ L. Nicastro, ${ }^{12}$ E. Palazzi ${ }^{12}$

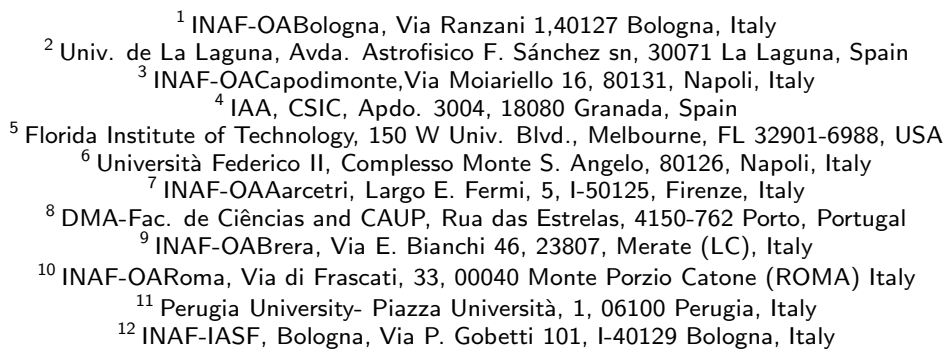

\begin{abstract}
We present preliminary results of a photometric study devoted to the two Herbig Ae stars V346 Ori and PDS 2, based on data from the R.E.M. telescope. As a result, 1) we confirm the multiperiodicity of V346 Ori; 2) we discover $\delta$ Scuti-like pulsation in PDS 2.
\end{abstract}

\section{Introduction}

V346 Ori and PDS 2 are interesting objects: V346 Ori was already suspected to be a multiperiodic PMS $\delta$ Scuti star (Pinheiro et al. 2003), whereas PDS 2 was investigated because its spectral type F3V makes it a very good object to constrain the poorly sampled red edge of the PMS $\delta$ Scuti instability strip. We studied these two objects by using the $0.6 \mathrm{~m} \mathrm{R}$.E.M. telescope (La Silla, Chile, www.rem.inaf.it). Note that present R.E.M. observations for V346 Ori are part of a multisite campaign for which data analysis is ongoing.

\section{Results}

Due to the lack of space, here we only present the periodogram obtained for V346 Ori (see Fig. 1) based on about $94 \mathrm{~h}$ of R.E.M. observations. These data allowed us to identify at least 9 significant frequencies (see figure). A similar analysis for PDS 2, ( $22 \mathrm{~h}$ of R.E.M. observations during 7 nights), allowed us to establish that PDS 2 is a multiperiodic pulsating star with at least three significant oscillation frequencies at $f 1=17.05 \mathrm{c} / \mathrm{d}, \mathrm{f} 2=13.77 \mathrm{c} / \mathrm{d}$, $\mathrm{f} 3=24.24 \mathrm{c} / \mathrm{d}$. Thus, PDS 2 is a new member of the PMS $\delta$ Scuti class. In the future we will: 1) finalize the analysis for V346 Ori (taking advantage of the photometry from other sites) and for PDS 2; 2) interpret the periodicities found for the two stars in the light of both radial and non-radial pulsation models.

\section{References}

Pinheiro F. J. G., Folha D. F. M., Marconi M., et al., 2003, A\&A, 399, 271 


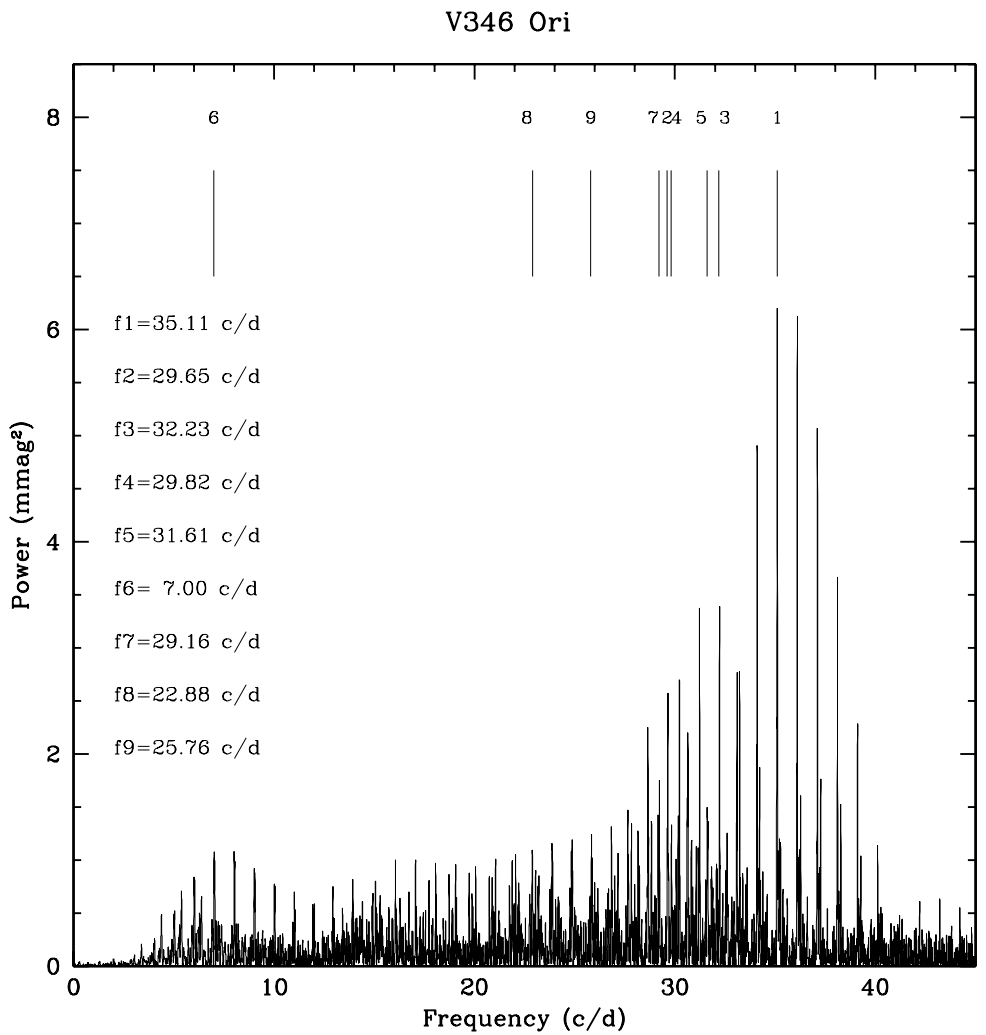

Figure 1: Periodogram for V346 Ori R.E.M. data. All significant frequencies are indicated and labelled. 